\title{
A Rule-Based Approach to Prepositional Phrase Attachment Disambiguation
}

\author{
Eric Brill \\ Spoken Language Systems Group \\ Laboratory for Computer Science, M.I.'T. \\ Cambridge, Ma. 02139 U.S.A \\ brill@goldilocks.lcs.mit.edu \\ Philip Resnik* \\ Sun Microsystems Laboratorics, Inc. \\ Chelmsford, MA 01824-4195 U.S.A \\ philip.resnik@east.sun.com
}

\begin{abstract}
In this paper, we describe a new corpus-based approach to prepositional phrase attachment disambiguation, and present results comparing performance of this algorithm with other corpus-based approaches to this problem.
\end{abstract}

\section{Introduction}

Prepositional phrase attachment disambiguation is a difficult problem. Take, for example, the sentence:

(1) Buy a car [ $r p$ with a steering wheel].

We would guess that the correct interpretation is that onc should buy cars that como with stcering wheels, and not that one should use a steering wheel as barter for purchasing a car. In this case, wo are helped by our world knowledge about antomobiles and automobile parts, and about typical methods of barter, which we can draw upon to correctly disambiguate the sentence. Beyond possibly needing such rich semantic or conceptual information, Altmann and Steedman (AS88) show that there are certain cases where a discourse model is needed to correctly disambiguate prepositional phrase attachment.

However, while there are certainly cases of ambiguity that seem to need some deep knowledge, either linguistic or conceptual, one might ask what sort of performance conld be achieved by a system that uses somewhat superficial knowledge an-

\footnotetext{
"Parts of this work done at the Computer and Information Science Department, University of Pennsylvania were supported by by DARPA and AFOSR jointly under grant $N_{0}$. AlFOSR-90-0066, and by AlRO grant No. DAA a 03-89-C003.1 l'RI (first author) and by an IBM graduate fellowship (second anthor). This work was also supported at MIT by ARPA under Contract N00014-89-J-1332, monitored through the Office of Naval research (first anthor).
}

tomatically xtracted from a large corpus. Recent work has shown that this approach holds promise (ITR91, HR93).

In this paper wo describe a new rule-based approach to prepositional phrase attachment risambiguation. A set of simple rules is learned aur tomatically to try to predict proper attachment based on any of a number of possible contextual cues.

\section{Baseline}

Hindle and Rooth (ITR91, HR93) describe a corpus-based approach to disambiguating between prepositional phrase attachment to the main verb and to the object noun phrase (such as in the example sentence above). They first point ont that simple attachmont strategies such as right associattion (Kim73) and minimal attachment (Fra78) do not work well in practice (sce (WFB90)). They then suggest using lexical preference, estimated from a large corpus of text, as a method of resolving altachment ambiguity, a technique they call "lexical association." From a large corpus of parsed text, they first find all noun phrase heads, and then record the verb (if any) that precedes the head, and the preposition (if any) that follows it, as well as some other syntactic information abont the sentence. An algorithm is then spocified to try to extract attachment information from this table of co-occurrences. For instance, a table entry is considered a definite instance of the prepositional phrase attaching to the noun if:

'L'he noun phrase occurs in a context where no verb could license the prepositional phrase, specifically if the nom plirase is in a subject or: other pre-verbal position.

'They specify seven different procedures for deciding whether a table entry is an instance of no attachment, sure noun attach, sure verb attach, or an ambiguous attach. Using these procedures, they are able to extract frequency information, 
counting the number of times a particular verb or noun appears with a particular preposition.

These froquencies serve as training data for the statistical model they use to predict correct attachmont. To disanbiguate sontence (1), they would compute the likelihood of the preposition with given the verb tuy, and contrast that with the likelihood of that preposition given the noum wheel.

One problem with this approach is that it is limited in what rolationships are examined to make an attachment decision. Simply oxtending Findle and Rooth's model to allow for relationships such as that between the verb and the object of the preposition would result in too large a paramotor space, given any roalistic quantity of training data. Another problem of the nethod, shared by many statistical approaches, is that the model acquired dnring training is represented in a huge table of probalsilities, precluding any straightforward analysis of its workings

\section{Transformation-Based Error-Driven Learning}

Transfomation-based error-driven learning is it simple learning algorithm that has been ipplocel to a number of natural langhage problens, inclusting part of speech tagging and syntactic parsing (Bri92, Bri93a, Bri93b, Bri94). ligure 1 illustrates the leaning process. First, unanotated text is passed through tho initial-state annotia tor. 'The initial-state annotator can range in complexity from quite trivial (e.g. assigning rantom structure) to quite sophisticated (e.g. assigning the outiput of a knowledge-based annotator that was created by hand). Once text has been passed througl the initial-state annotator, it is then compared to the truth, as indicated in a mamally annotatied corpus, and transformations are tearned that can be applied to the outpul of the initial state annotatior to malio it better resemble the truth.

So far, only a greoly search approach lias been used: at each iteration of learning, he transfornation is found whose application results in the greatest improvement; that transformation is then added to the ordered transformation list, and the corpus is updated by applying the learned transformation. (See (RM9l) for a detailed discussion of this algorithm in the context of machine learning issues.)

Once an ordered list of transformations is learned, now text can be annotated hy first applying the initial state annotator: to it and then applying each of the transformations, in order.

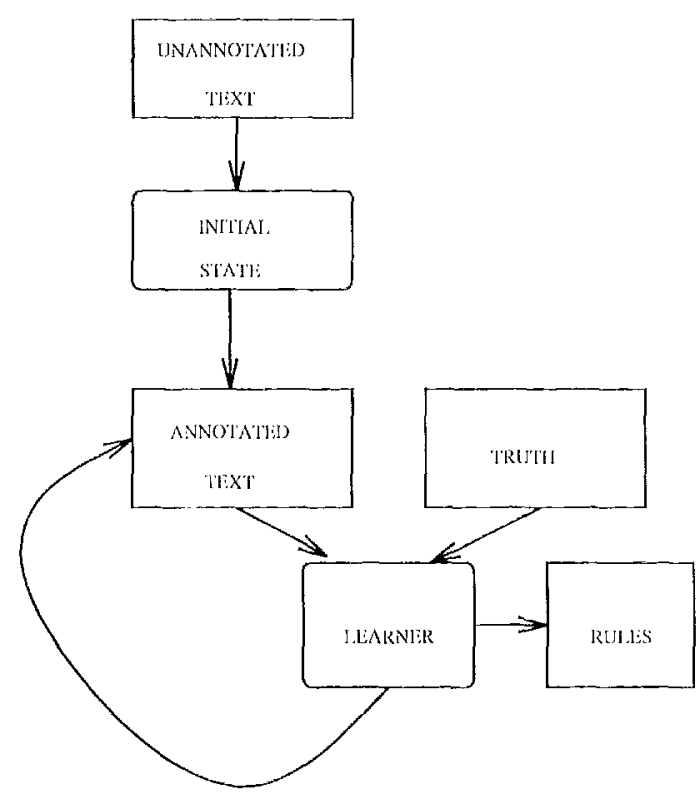

Figure 1: Transfornation-Based Intor-1)riven Iarming.

\section{Transformation-Based Prepositional Phrase Attachment}

We will now show how thansformation-based errordriven learning can be used to resolve prepositional phrase at tachment ambiguity. 'T'he preposilional phrase attachmont barner learus transformations from at corjus of A-tuples of the form (v $n \perp 1, n 2$ ), where $v$ is a verb, $n 1$ is the head of its object noun phase, $p$ is the preposition, and 112 is the lacad of the noum phrase governed by the preposition (for cxample, see/o the boy/nt on $/ \mathrm{p}$ the hill/ug). lior all sentences that conform to this pattern in the Ponn Trechank Wall Street Journal corpus (MSM93), such a 4-tuple was formed, and each d-tuple was paired with the attachment decision used in the Treobank parse." 'There were 12,7664 -tuples in all, which were randomly split into 12,266 training samples and 500 tost samples. In this experiment (as in (IIR91, HL93)), the attachment choice for prepositional plurases was be1ween the object nom and the matrix verb. Tu the initial stiate amnotator, all prepositional phrasos

\footnotetext{
'Patterus were extracted using tgrep, a tree-bascel grep program written by likeh Pito. Thlo 4-tuples woro cxtracted antomatically, and mistakes wero not manually pruned ont.
} 
are attached to the object noun. ${ }^{2}$ 'This is the attachment predicted by right association (Kim73).

The allowable transformations are described by the following templates:

- Change the attachment location from X to $\mathrm{Y}$ if:

- $\mathrm{n} 1$ is $W$

$-\mathrm{n} 2$ is $W$

- $v$ is $W$

- $p$ is $W$

$-\mathrm{n} 1$ is $W 1$ and $n 2$ is $W 2$

$-n 1$ is $W 1$ and $v$ is $W 2$

$-\ldots$

Here "from $\mathrm{X}$ to $\mathrm{Y}$ " can be either "from $\mathrm{n} 1$ to $\mathrm{v}$ " or: "from v to $n 1, " W(W 1, W 2$, etc.) can be any word, and the ellipsis indicates that the complete set of transformations permits matching on any combination of values for $v, n 1, p$, and $n 2$, with the exception of patterns that specify values for all four. For example, one allowable transformation would be

Change the attachment location from $\mathrm{n} 1$ to $\mathrm{v}$ if $p$ is "until".

Learning proceeds as follows. First, the training set is processed according to the start state annotator, in this case altaching all prepositional phrases low (attached to n1). Then, in essence, each possible transformation is scored by applying it to the corpus and computing the reduction (or increase) in error rate. In reality, the search is data driven, and so the vast majority of allowable transformations are not examined. The best-scoring transformation then becomes the first transformation in the learned list. It is applied to the training corpus, and learning continues on the modified corpus. This process is iterated until no rule can be found that reduces the error rate.

In the experiment, a total of 471 transformations were learned - Figure 3 shows the first twenty. ${ }^{3}$ Initial accuracy on the test set is $64.0 \%$ when prepositional phrases are always attached to the object noum. After applying the transformations, accuracy increases to $80.8 \%$. Figure 2 shows a. plot of test-set accuracy as a function of the number of training instances. It is interesting to note that the accuracy curve has not yet reached a

${ }^{2}$ If it is the case that attaching to the verb would be a better start state in some corpora, this decision could be parameterized.

${ }^{3}$ In transformation $\# 8$, word token amount appears because it was used as the head noun for noun phrases representing percentage amounts, e.g. "5\%." The rule captures the very regular appearance in the Penn Tree bank Wall Street Journal corpus of parses like Sales for the year [VP rose [NP $5 \%$ J $P P$ in fiscal 1988]].

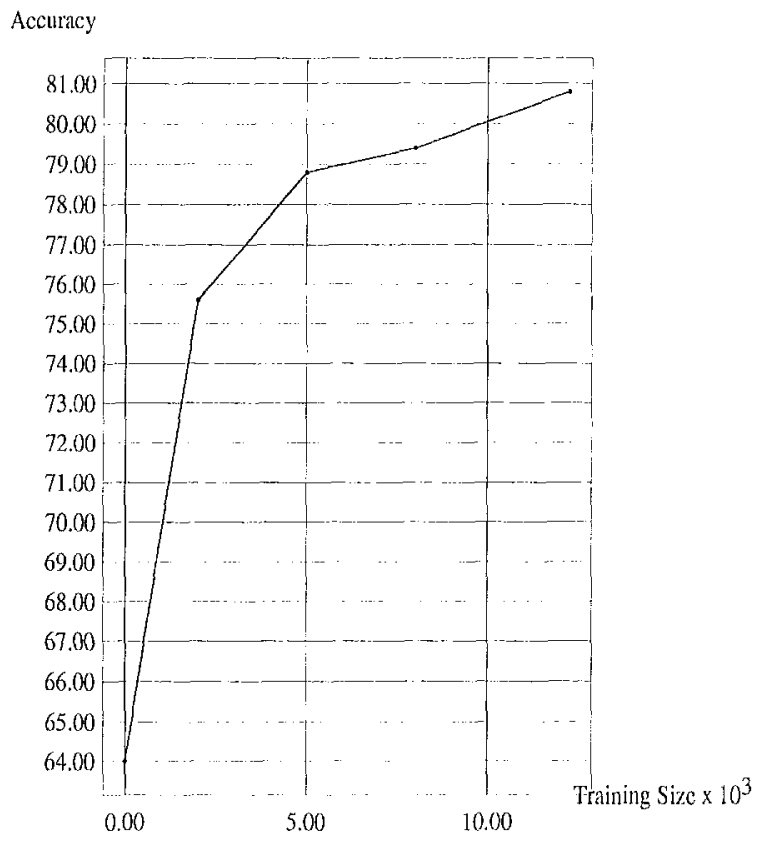

Figure 2: Accuracy as a function of training corpus size (no word class information).

plateau, suggesting that more training data would lead to further improvements.

\section{Adding Word Class Information}

In the above experinent, all transformations are triggered by words or groups of words, and it is surprising that good performance is achieved even in spite of the inevitable sparse data problems. There are a number of ways to address the sparse data problem. One of the obvious ways, mapping words to part of speech, seens unlikely to help. Instead, semantic class information is an attraclive alternative.

We incorporated the idea of using scmantic information in the following way. Using the WordNet noun hierarchy (Mil90), each noun in the training and test colpus was associated with a set containing the noun itself plus the name of every semantic class that noun appears in (if any). ${ }^{4}$ The transformation template is modified so that in addition to asking if a noum matches some word $W$,

\footnotetext{
${ }^{4}$ Class names corresponded to unicue "synonym set" identifiers within the WordNet noun database. A noun "appears in" a class if it falls within the hyponym (IS- $\Lambda$ ) tree below that class. In the experiments reported here we used WordNet version 1.2.
} 


\begin{tabular}{|c|c|c|c|}
\hline \multirow[b]{2}{*}{$\#$} & \multicolumn{2}{|c|}{$\begin{array}{l}\text { Change Attachnent } \\
\text { location }\end{array}$} & \multirow[b]{2}{*}{ Condition } \\
\hline & From & lo & \\
\hline 1 & N1 & V & $P$ is al \\
\hline 2 & N1 & V & P'is as \\
\hline 3 & NI & V & $P$ is into \\
\hline 1 & NI & V & $\mathrm{P}$ is from \\
\hline 5 & $\mathrm{~N} 1$ & V & $\mathrm{P}$ is with \\
\hline 6 & N1 & $\bar{V}$ & $\mathrm{~N} 2$ is year \\
\hline 7 & N1 & V & $P^{\prime}$ is $b y$ \\
\hline 8 & N1 & V & $\begin{array}{l}P \text { is in and } \\
N 1 \text { is amount }\end{array}$ \\
\hline 9 & N1 & V & P' is through \\
\hline 10 & N1 & V & $\mathrm{P}$ is during \\
\hline 11 & N1 & $\vec{V}$ & $V$ is $m u t$ \\
\hline 12 & $\bar{N} 1$ & $V^{-}$ & $\mathrm{N2}$ is month \\
\hline 13 & N! & V & $P$ is under \\
\hline 14 & $\mathrm{~N} \perp$ & $\bar{V}$ & P' is aller \\
\hline 15 & N1 & V & $\begin{array}{l}V \text { is have and } \\
P \text { is in }\end{array}$ \\
\hline 16 & $\mathrm{NL}$ & V & $P$ is withoul \\
\hline 17 & $\mathrm{~V}$ & NI & Pis of \\
\hline 18 & $\mathrm{NL}$ & V & $\begin{array}{l}V \text { is buy and } \\
P^{\prime} \text { is for }\end{array}$ \\
\hline 19 & $\mathrm{N1}$ & V & $\mathrm{P}$ is before \\
\hline 20 & $\mathrm{~N} . \mathrm{I}$ & V & $\begin{array}{l}V \text { in have and } \\
P \text { is on }\end{array}$ \\
\hline
\end{tabular}

l'igure 3: The first 20 transformations learned for prepositional phrase attachmont. it can also ask if it is a member of some class C." 'This approach to data sparseness is similar to that of (Res93b, Rill93), where a method is proposed for using Word Net in conjunction with a corpus to obtain class-based statistics. Our method here is much simpler, however, in that wo are only using Boolean valuos to indicate whether a word can be a merrber of a class, rather: than estimating a full set of joint probabilitics involving classes.

Since the transformation-based approach with classes can gencralize in a way that the approach without classes is mable to, wo would expect fewer transformations to be necessary. Experimentally, this is inderd the case. In a second experiment, training and testing were carried out on the same samples as in the previous experiment, but this time using the extended transformation templatios for word classes. A total of 260 transformations were learned. Applying these transformations to the tost set resulted in an accuracy of $81.8 \%$. In figure 4 we show the lirst 20 transformations learned using nom classes. Class descriptions are surounded by square brackets. "The first transformation states that if $\mathrm{N}^{2}$ is a nom that describes time (i.e. is a member of WordNet class that includes the nouns "yoar," "month," "week," and others), then the prepositional phrase should be altiached to the verb, since tine is much more likely to modily a verb (e.g. leane the meeting in an hour) than a noun.

This experiment also demonstrates low any fealure-based lexicon or worel classification schene can trivially be incorporated into the learner, by extending transformations to allow them to make reference to a word and any of its features.

\section{Fvaluation against Other Algorithms}

In (IRR91, MR93), training is clone on a superset of sentence types used in traininty the transformation-based leaner. 'The transtormation-based learner is trained on sentences containing $\mathrm{v}, \mathrm{nl}$ and $\mathrm{p}$, whereas the algorithm described by IIindle and Rooth can also use? sentences containing only $\mathrm{v}$ and $p$, or only $n 1$ and p. In their paper, they train on over $200,000 \mathrm{sen}-$ tences with prepositions from the Associaled Press $(A P)$ newswire, and they quote an accuracy of 78 $80 \%$ on $A P$ tiest diata.

\footnotetext{
5 For reasons of rutt-time efliciency, transformations making reference to the classes of both $n 1$ and $n 2$ were not permitted.

Gor expository parposes, the unique WordNet, identifiers have been replaced by worls that describe the content of the class.
} 


\begin{tabular}{|c|c|c|}
\hline Mothod & Accuracy & $\begin{array}{c}\text { \# of } \\
\text { Transforms }\end{array}$ \\
\hline \hline t-Scores & $70.4-75.8$ & \\
\hline Thansformations & 80.8 & 171 \\
\hline $\begin{array}{c}\text { Transformations } \\
\text { (no N2) }\end{array}$ & 79.2 & 118 \\
\hline $\begin{array}{c}\text { Transformations } \\
\text { (classes) }\end{array}$ & 81.8 & 266 \\
\hline
\end{tabular}

\begin{tabular}{|c|c|c|c|}
\hline \# & $\begin{array}{l}\text { Cha } \\
\text { Attact } \\
\text { Jocat } \\
\text { From }\end{array}$ & $\begin{array}{l}\text { ge } \\
\text { nent. } \\
\text { ion } \\
\text { 'To }\end{array}$ & Conclition \\
\hline 1 & N1 & $\bar{V}$ & $\mathrm{~N} 2$ is [time] \\
\hline 2 & N1. & V & $\mathrm{P}$ is $a t$ \\
\hline 3 & N1 & V & $\mathrm{P}$ is as \\
\hline 4 & N1 & V & $P$ is into \\
\hline 5 & NI & V & $\mathrm{P}$ is from \\
\hline 6 & N1 & $\mathrm{V}$ & $\mathrm{P}^{3}$ is with \\
\hline 7 & N1 & $\bar{V}$ & $\mathrm{P}$ is of \\
\hline 8 & N1 & V & $\begin{array}{l}\mathrm{T} \text { is in and } \\
\text { NI is } \\
\text { [measure, quantity, amount] }\end{array}$ \\
\hline 9 & N1 & V & $\begin{array}{l}\mathrm{P} \text { is by and } \\
\mathrm{N} 2 \text { is [abstraction] }\end{array}$ \\
\hline 10 & N1 & V & $\mathrm{P}$ is through \\
\hline 11 & N1 & V & $\begin{array}{l}\mathrm{P} \text { is in and } \\
\mathrm{N} 1 \text { is } \\
\text { [group, grouping] }\end{array}$ \\
\hline 12 & $\mathrm{~V}$ & N1 & $V$ is be \\
\hline 13 & NI & $\bar{V}$ & $\mathrm{~V}$ is put \\
\hline 14 & N1 & V & $P$ is under \\
\hline 15 & N1 & V & $\begin{array}{l}\mathrm{P} \text { is in and } \\
\mathrm{N} 1 \text { is } \\
\text { [uritten communication] }\end{array}$ \\
\hline 16 & $\mathrm{Ni}$ & $\mathrm{V}$ & '? is without \\
\hline 17 & N1 & V & $P$ is during \\
\hline 18 & N1 & $\mathrm{V}$ & $\begin{array}{l}\mathrm{P} \text { is on and } \\
\mathrm{N} 1 \text { is [thing] }\end{array}$ \\
\hline 19 & N1 & V & $\mathrm{P}$ is after \\
\hline 20 & N1 & V & $\begin{array}{l}\mathrm{V} \text { is buy and } \\
\mathrm{P} \text { is for }\end{array}$ \\
\hline
\end{tabular}

Tigure 4: 'The first 20 transformations learned for prepositional phrase attachment, using noun classes.
Figure 5: Comparing Results in PP Attachnent.

In order to compare the two approaches, we reimplemented the algorithm from (IIR,91) and tested it using the same training and tost set used for the above experiments. Doing so resulted in an attachment accuracy of $70.1 \%$. Next, the training set was expanded to include not only the cases of ambiguous attachment found in the parsed Wall Street Journal corpus, as before, but also all the unambiguous prepositional phrase attachments found in the corpus, as well (continuing to cxclude the test set, of course). Accuracy improved to $75.8 \%{ }^{7}$ using the larger training set, still significantly lower than accuracy obtained using the transformation-based approach. The technique described in (Res93b, RH93), which combined Hindle and Rooth's lexical association technique with a WordNet-based conceptual association measure, resulted in an accuracy of $76.0 \%$, also lower than the results obtained using transformations.

Since Hindle and Rooth's approach does not make reference to $n 2$, we reran the transformation-learner disallowing all transformations that make reference to n2. Doing so resulted in an accuracy of $79.2 \%$. Sce figure 5 for a summary of results.

It is possible to compare the results described here with a somewhat similar approach developed independently by Ratnaparkhi and Ronkos (RR94), since they also used training and test data drawn from the Penn 'Treebank's Wall Strect Jonrnal corpus. Instead of using manually constructed lexical classes, they use word classes arrived at via mutual information clustering in a training corpus $\left(13 \mathrm{D} \mathrm{d}^{+} 92\right)$, resulting in a representation in which each word is represented by a sequence of bits. As in the experiments here, their statistical model also makes use of a 4-tuple context ( $v, n 1, p, n 2)$, and can use the identities of the words, class information (for them, values of any of the class bits),

\footnotetext{
The difference between these results and the result they quoted is likely due to a much larger training set used in their original experiments.
} 
or both linds of iuformation as contextual fat tures they describe a search process used to determine what subset of the available information will be ased in the mortel. Given a choice of features, they train a probabilistic model for Pr(Sitc|context), and in testing choose Site ... v or Site $=11$ according to whele has the higher conclitional probability.

Ratnaparkhi and Roukos report, an atcentacy of $81.6 \%$ using both word and chass information on Wall Strect Journal text, using a traming corpus twice as large as that used in our experiments. They also report that a decision tree model constructed using the same features and training datia achieved performance of $77.7 \%$ on the sane test sel.

$A$ nunber of other researchers have aplored corpus-based approaches to prepositional phrase al tachment disambiguation that, make use of word dasses. J'or example, Weischodel al al. (WAI'+91) and Basili et al. (BPY9I) both describe the use of namually constructed, clomain specific word classes together with corpus-based statisties in order to resolve prejositional phrase at tachnent ant. biguity. Because these papers describe results ol)tained on different corpora, however, it is difficult to make a performánce comparison.

\section{Conclusions}

The transformation-based approach to resolving prepositional phrase disanubignation has a number of advantages over other approaches. Th a direct. comparison with lexical association, higher accaracy is achieved using words alone even though abtachment information is captured in a relatively small number of simple, readable rules, as opposed to a large munber of lexical co-oecurrenes probabilities.

In addition, we have shown how the transformation-based learner can casily be ex. tended to incorporate word-class information. 'This resulted in a shight incroase in pertormance, but more notably it resulted in a reduction by roughly half in the total $11 u m b e r$ of transformation rules needed. And in contrast to approaches using class-based probabilistic models (BPV91, Res93c, WA13+01) or classes derived via statistical clustering metlods (RROA), this technicue produces a rule set that captures conceptual generalizations concisely and in human-readable form.

Furthomore, insofar as comparisons can be makle anong separate experiments using Wall Street Jourual training and test datia ((TIR.91), reimplenented as reported above; (Res93c,
RHO3); (RR94)), the rulc-based approach de scribed here achieves better performance, using ar algorithm that is conceptaally quite simple and in practical terms extremely casy to implement.

$A$ more general point is that the transformalion-based approach is easily adapled to situations in which some loarning from a corpus is desirable, lut hand-constructed prior knowledge is also available. lixisting knowlelge, such is structural strategies or even a priort lexical preferences, can be incorposated into tho start state annotator, so that the learning algorilhn begins with more refined input. And known exceptions can be handled tansparently simply by atding additional mules to the set that is learned, using the sance representation.

$\Lambda$ disadvantage of the approncl is that it requires supervised training - that is, a representative set of "true" cases from which to leam. However, this becomes less of a problem as anmotiated corpora become increasingly available, and suggests the combination of supervised and unsupervised methods as an intoresting avenue for further research

\section{References}

[AS88] C. Altmann and M. Stcodnan. Interaction with context during human sentience procossing. Cognition, 30:191. $238,1988$.

[BDd+92] Peter l'. Brown, Vincent J. Della P'ietra, Peter V. desouza, Jennifer C. Lai, and Robert h. Mercer. Classhased n-gran models of natural lint. guage. Compulational linguistics, 18(4):467 480, Decomber 1992.

[131'V91] R. Basili, M. Parien\%a, and J'. Volardi. Combining NIsl' and statistical techniques for lexical acquisition. In Proceedings of the AAAl Fall Symposium on Probabilistic Approaches to Natural language, Cambridgo, Massachusetis, October 1991.

[Brig2] F. Brill. A simple rule-besed part of speccli lagger. In Proccedings of the Third Conference on Appliod Nalural Tanguage Processing, $A C$ C, Trento, tialy, 1992.

[13ri93a] Jis Brill. Automatic grammar induction and parsing free text: $\Lambda$ transformation-based approach. In Procecdings of the 3tst Meeting of the Association of Computational linguistics, Columbus, Olı, 1993.

\footnotetext{
"Our code is being made publicly available. Contaret the anthors for information on how to obtain it.
} 
[Bri93b] E. Brill. A Corpus-Based Approach to Language learning. P'hD thesis, Department of Compater and Information Science, University of Penusylvania, 1993.

[Bri94] F. Brill. Some advances in rule-based part of speech tagging. In Proceedings of the T'uelfth National Conference on Artificial Intelligence (AAA91), Scattlc, Wa.., 1994.

[Tra78] I. lirazier. On comprehending sentences: syntactic parsing strategies. PhD thesis, University of Comnecticut, 1978.

[HR91] D). Findle and M. Rooth. Structural ambiguity and lexical relations. In Procecdings of the 29th Annual Meeting of the Association for Computational Linguistics, Berkeley, Ca., 1991.

[HR93] 3. Hindle and M. Rooth. Structural ambiguity and lexical relations. Computational Linguistics, 19(1):103-120, 1993 ,

[Kim73] J. Kimball. Seven principles of surface structure parsing in natural lauguage. Cognition, 2, 1973.

[Mil90] G. Miller. Wordnet: an on-line lexical database. International Journal of Lexicography, 3(4), 1990.

[MSM93] M. Marcus, B. Santorini, and M. Marcinkiewicz. Building a large annotated corpus of English: the Pem Treebank. Computational Linguistics, 19(2), 1993.

[Res93a] P. Resnik. Selcclion and Information: A Class-Based Approach to Lexical Relationships. PhD thesis, University of Pennsylvania, Jecember 1993. (Institute for Research in Cognitive Science report IRCS-93-42).

[Res)3b] P. Resnik. Semantic classes and syntactic ambiguity. In Proceedings of the ARPA Workshop on Human Language Tcchnology. Morgan Kaufman, 1993.

[Res93c] P. Resnik. Semantic classes and syntactic anbiguity. ARPA Workshop on IIuman Language Technology, March 1993. Princeton.

[RH93] P. Rosnik and M. Hearst. Syntactic ambiguity and conceptual relations. In K. Church, editor, Procecdings of the ACl Workshop on Very Large Corpora, pages 58-64, June 1993.
[RM94] I. Ramshaw and M. Marcus. Exploring the statistical clerivation of transformational rule sequences for part-ofspeech tagging. In J. Klavans and P. Resnik, editor's, The Balancing Act: Proceedings of the ACL Workshop on Combining Symbolic and Statistical Approaches to Language, New Mexico State University, July 1994.

[RR94] A. Ratnaparkhi and S. Ronkos. A maximum entropy model for prepositional phrase attachment, In Proceedings of the ARPA Workshop on Human Language Technology, PlainsLoro, NJ, March 1994.

[WA $3+9$ 1] R. Weischedel, D, Ayuso, R. Bobrow, S. Boisen, R. Ingria, and J. Palmucci. Partial parsing: a report of work in progress. In Proceedings of the liourth DARPA Speech and Natural hanguage. Workshop, February 1991, 1991.

[WrB90] G. Whittenore, K. Ferrara, and H. Brumer. Empirical study of predictive powers of simple attachment schemes for post-modifier prepositional phrases. In Proceedings of the 28th Annual Mecting of the Association for Compulational Linguistics, 1.990. 